\title{
A COMPARATIVE STUDY ON 2-PORT VERSUS 3-PORT LAPAROSCOPIC APPENDICECTOMY
}

\author{
Paatneedi Naresh Kumar1, Ravula Mahalakshmi², Gonugunta Sri Rangasai Karthik ${ }^{3}$
}

${ }^{1}$ Associate Professor, Department. of General Surgery, Rangaraya Medical College and Govt. General Hospital, Kakinada, Andhra Pradesh.

2Professor, Department of General Surgery, Rangaraya Medical College and Govt. General Hospital, Kakinada, Andhra Pradesh. 3 Postgraduate Student, Department of General Surgery, RMC, Kakinada.

\section{BACKGROUND}

\section{ABSTRACT}

Appendicitis is the most common of the surgically managed pain abdomen cases. Early surgical intervention improves outcome. First laparoscopic appendectomy was performed by deKok in 1977.1 Laparoscopic appendicectomy is the standard of treatment and has standard advantage over open appendicectomy ${ }^{2}$ with a slight increase in formation of intra-abdominal abscess. ${ }^{3}$

The aim was to study the duration of anaesthesia, postop analgesic doses required, duration of hospital stay and complication profile of standard 3-port appendectomy vs. 2-port lap. assisted appendectomy in cases with early appendicitis.

\section{MATERIALS AND METHODS}

A prospective study was conducted in Government General Hospital, Kakinada. The study period is from 1 st June 2016 to $15^{\text {th }}$ October 2016. The study sample is 62 patients, randomised to both groups in patients with acute appendicitis with no adhesions or mass formation.

\section{RESULTS}

There is decrease in the duration of patient under anaesthesia, number of postop analgesic doses required, duration of hospital stay and complication profile was studied and found be better than that with standard 3-port appendectomy.

\section{CONCLUSION}

In patients with early appendicitis with no mass formation or adhesions 2-port lap. assisted open appendicectomy can be done which decreases the duration of patient under anaesthesia, postoperative pain and with less number of postoperative doses of analgesic required. It helps in early enteral feeding, early mobilisation and early discharge of patient.

\section{KEYWORDS}

2-Port Appendectomy, Lap. assisted Open Appendectomy, Lap. Appendectomy with Decreased Postop Morbidity.

HOW TO CITE THIS ARTICLE: Kumar PN, Lakshmi RM, Karthik GSRS. A comparative study on 2-port versus 3-port laparoscopic appendicectomy. J. Evolution Med. Dent. Sci. 2016;5(103):7545-7546, DOI: 10.14260/jemds/2016/1708

\section{BACKGROUND}

A study was conducted in 62 patients suffering with early acute appendicitis. Study of 2-port versus 3-port laparoscopic appendicectomy was done and duration of anaesthesia, duration of hospital stay, postop analgesia required and mobilisation of patient, port site infections are studied.

\section{Procedure}

In patients with clinical and radiological findings suggestive of acute appendicitis, laparoscopic appendicectomy was planned and under general anaesthesia, a 10 -mm supraumbilical port is placed and pneumoperitoneum is created. Another $10-\mathrm{mm}$ port is placed in the right iliac fossa and mobility and adhesions of appendix is tested for. All the patients with mass formation or adhesions are left out of study and conventional 3-port technique was used. In those patients with early

Financial or Other, Competing Interest: None.

Submission 14-10-2016, Peer Review 30-11-2016,

Acceptance 07-12-2016, Published 26-12-2016.

Corresponding Author:

Dr. Paatneedi Naresh Kumar,

\#10-5-27/1, Nageswar Rao Street,

Ramarao Peta,

Kakinada, Andhra Pradesh.

E-mail: drnaresh180532@yahoo.com

DOI: $10.14260 /$ jemds/2016/1708 appendicitis, base of the appendix is marked with diathermy to prevent leaving a longer stump, and through the RIF port appendix is delivered out with the port around by reducing the pneumoperitoneum, mesoappendix is divided as in open method and appendix is removed. Wound wash is given. Camera is reintroduced through the umbilical port and haemostasis is confirmed and standard port closure is done.

\section{Inclusion Criteria}

Patients suffering from appendicitis and USG suggestive of acute appendicitis in patients with long mesoappendix.

\section{Exclusion Criteria}

Those patients where USG was suggestive of appendicular mass formation.

On introducing the camera port, if mass formation or dense adhesions are observed, 3-port technique is used.

\section{RESULTS}

Of the 62 patients with early appendicitis studied, randomisation was done and 28 patients were selected for 2port appendicectomy and 34 underwent 3-port appendicectomy.

Duration of anaesthesia, Duration of surgery was calculated from insertion of umbilical port to the time of port closure. 


\begin{tabular}{|c|c|c|}
\hline $\begin{array}{c}\text { Time } \\
\text { Duration }\end{array}$ & $\begin{array}{c}\text { 2-Port } \\
\text { Technique }\end{array}$ & $\begin{array}{c}\text { 3-port } \\
\text { Technique }\end{array}$ \\
\hline$<20$ min. & 24 & 16 \\
\hline$>20$ min. & 4 & 18 \\
\hline \multicolumn{2}{|c|}{ The Mean Duration was about 18 minutes } \\
Compared to 26 minutes in 3-port Technique \\
\hline
\end{tabular}

\begin{tabular}{|l|c|}
\hline & $\begin{array}{c}\text { Mean Duration of Stay in } \\
\text { Days }\end{array}$ \\
\hline In 2-port technique & 1.6 \\
\hline In 3-port technique & 2.2 \\
\hline \multicolumn{2}{|c|}{ Duration of Hospital Stay } \\
\hline
\end{tabular}

\begin{tabular}{|c|c|}
\hline & $\begin{array}{c}\text { Mean No. of Doses of } \\
\text { Parenteral Analgesics Needed }\end{array}$ \\
\hline 2-port technique & 2.2 \\
\hline 3-port technique & 3.6 \\
\hline \multicolumn{2}{|c|}{ Doses of Postoperative Analgesia } \\
\hline
\end{tabular}

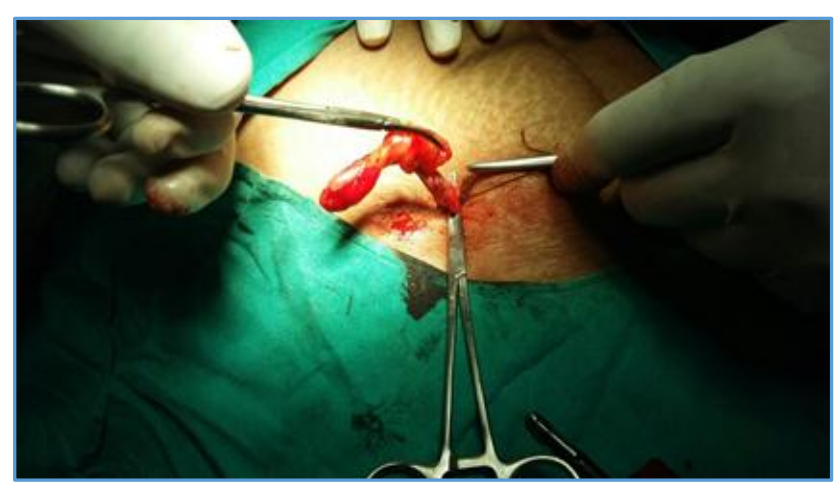

Port Site Infections: Only 1 Case had Port Site Infection Requiring Prolonged Parenteral Antibiotics

\section{DISCUSSION}

In patients who present with early acute appendicitis, a 2-port lap. assisted open appendicectomy can be done after ruling out any mass formation by introducing the scope from the umbilical camera port.

This decreased the duration of surgery in lap. assisted open technique - $18 \mathrm{~min}$., whereas in a standard technique it was about 26 min., the major difference in the operative duration was due to knotting of base of appendix either by endoknotting or by extracorporeal knotting. Decrease in the operative period decreased the patient's duration under anaesthesia. Yagnik et al $^{4}$ studied that operative time was significantly lower $(P=0.03)$ in 2-port and significantly higher $(P=0.001)$ in 3-port technique as compared to open appendectomy. Khan and Al-Bassam 5 did 2-port LA on 45 patients and 3-port LA on 41 patients and found that 2-port LA was quicker to perform.
In patients with early appendicitis with no signs of peritoneal contamination or mass formation, we generally allow oral feeds after $8 \mathrm{hrs}$. post laparoscopic appendicectomy and the patient is discharged after 2 days of parenteral antibiotics with oral antibiotics to continue for next 3 days. The mean duration of hospital stay in lap. assisted open appendicectomy is 1.6 days and in standard 3-port appendicectomy was 2.2 days. By decreasing the hospital stay, the overall expense on the patient can also be decreased.

Postoperative analgesia was converted to oral analgesics as soon as patient is started on orals and parenteral supplementation of analgesics are given only on patient's need. In 2-port appendicectomy, the average number of parenteral analgesic doses needed is 2.2 compared to 3.6 in standard 3-port appendicectomy.

Patients were mobilised early and discomfort caused by 2 ports was less compared to the standard 3 ports and patients also accepted early oral feeds leading to reduced doses of postoperative analgesics needed and lessened hospital stay.

Only one patient had port site infection and patient had discharge from wound for 3 days which was managed by daily dressings and prolonged parenteral antibiotics.

\section{CONCLUSIONS}

In early appendicitis cases, 2-port technique of appendicectomy (Lap. assisted open appendicectomy) can decrease the duration of patient under anaesthesia, doses of postop analgesia required and duration of hospital stay with minimal or no risk of port site infection.

\section{REFERENCES}

1. De Kok HJ. A new technique for resecting the non-inflamed not-adhesive appendix through a mini-laparotomy with the aid of a laparoscope. Arch Chir Neerl 1977;29(3):1958.

2. Neugebauer EA, Sauerland S, Fingerhut A, et al. EAES guidelines for endoscopic surgery: twelve years evidence based surgery in Europe. Berlin, Heidelberg: Springer 2006:p387-9.

3. Towfigh S, Chen F, Mason R, et al. Laparoscopic appendectomy significantly reduces length of stay for perforated appendicitis. Surg Endosc 2006;20(3):495-9.

4. Yagnik VD, Rathod JB, Phatak AG. A retrospective study of two-port appendectomy and its comparison with open appendectomy and three-port appendectomy. Saudi J Gastroenterol 2010;16(4):268-71.

5. Khan AR, Al-Bassam A. Two-port versus three-port laparoscopic appendectomy in children with uncomplicated appendicitis. Paed Endosurg Innovative Tech 2002;6:255-60. 